\title{
The Effective Buckling Length on Numerical Study of Pipe-Sectioned Pier-Pile Integral Steel Structure
}

\author{
Takayuki Omori, Akira Kasai, Rei Kohara \\ Oversea Division, Oriental Consultants Co., Ltd, Tokyo, Japan \\ Email: ohmori@oriconsul.com
}

How to cite this paper: Omori, T., Kasai, A. and Kohara, R. (2017) The Effective Buckling Length on Numerical Study of Pipe-Sectioned Pier-Pile Integral Steel Structure. Open Journal of Earthquake Research, 6, 159-167.

https://doi.org/10.4236/ojer.2017.64009

Received: August 19, 2017

Accepted: September 17, 2017

Published: September 20, 2017

Copyright $\odot 2017$ by authors and Scientific Research Publishing Inc. This work is licensed under the Creative Commons Attribution International License (CC BY 4.0).

http://creativecommons.org/licenses/by/4.0/

cC) $\underset{\mathrm{EY}}{\mathrm{i}}$ Open Access

\begin{abstract}
Pier-Pile integral structures provide construction works with many environmental and landscape advantages. For example, the space required to construct these structures is smaller than that of other bridges due to the footing being removed, meaning that it is not necessity to greatly change the surroundings of these bridges. While there are environmental and landscape advantages, there are also a few demerits for the overall land-scape designs, including demerits in the design of this proposed structure which consists of relatively slender parts. This proposed structure has already been constructed in areas where possibility of a severe earthquake is low. However, some problems that have yet to be examined are related to the use of this proposed structure in areas where earthquakes are frequent. Lacking detailed studies of its behavior during severe earthquakes, it is currently difficult to construct these structures in Japan. Consequently, it is necessary to investigate in detail limited performance about compression and bending moment, and earthquake-resistant performance of these structures in order to resolve these problems. In this paper, It was clarified the relationship between the rigidity of the ground and the effective buckling length by buckling analysis and elasto-plastic finite deformation analysis. Moreover, it was proposed a simplified formula using a proposed characteristic value $\beta$ and several factors for analysis accuracy. A simplified formula would support to determine the effective buckling length to design the pier using the load-bearing capacity curve based on the slenderness ratio parameter.
\end{abstract}

\section{Keywords}

Pire-Pile Integral Steel Structures, Effective Buckling Length, Pipe Section 


\section{Introduction}

Pier-Pile integral structures provide construction works with many environmental and landscape advantages. For example, the space required to construct these structures is smaller because there are no footings, which avoids changing topography and protects the landscape. Some examples of the use of Pier-Pile integral structures include the pile bent pier bridge in Japan and the pedestrian bridge [1] in other parts of the world. Figure 1 shows Example of Pier-Pile integral structure. However, construction of these structures presents a few problems. In particularly, damaged areas cannot be identified after being subjected to a major earthquake. Therefore, it is currently difficult to construct these structures in Japan, a country where major earthquakes frequently occur. It is necessary to understand compression and bending behavior of these members, earthquake-resistant performance, and limit performance in detail in order to tackle these problems.

In accordance with the Japanese standards ([2]; herein after Japan Standard II), piers are designed based on the capacity stress obtained from the load-bearing capacity curve after designing the slenderness ratio parameter from the effective length derived from boundary condition. However, in the case of Pier-Pile integral structures, it is hard to decide the effective buckling length for calculating slenderness ratio parameter as the lower part of the pier is supported by springs. Although the effective buckling length can be calculated from the beam theory on the elastic floor, it is unsuited for practical design due to its inconvenience.

In addition, the load-bearing capacity curve of the Pier-Pile integral structure supported by the springs at the lower part is different from the application condition of the load-bearing capacity curve shown in the Japan Standard II, so its applicability is unclear.

In view of this background, this paper aims to clarify the relationship between the effective buckling length of the Pier-Pile integral structure and characteristic value $\beta$ specified in the Japanese standards ([3]; herein after Japan Standard IV).

In this paper, the aerial part and the underground part of the Pier-Pile integral structure are referred to as "pier" and "pile" respectively.

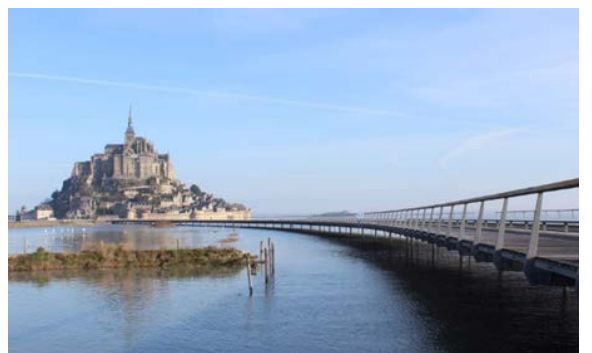

(a)

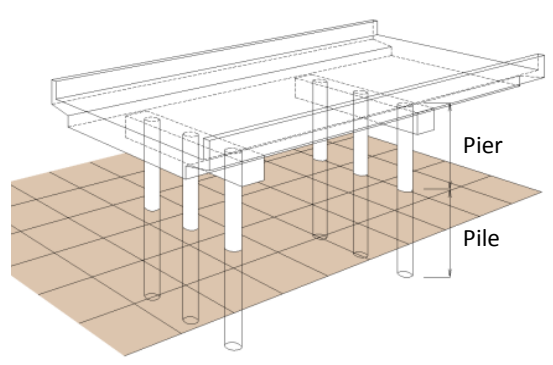

(b)

Figure 1. Example of Pier-Pile integral structure. (a) The pedestrian bridge; (b) The pile bent pier bridge. 


\section{The Target Structure of Study}

The target structure of this study is a single column pier with its lower part supported by spring. Figure 2 shows a single column pier with lower part supported by spring. It consists of a steel pipe section with an outer diameter of $D=500$ $\mathrm{mm}$, which is the standard size, and a thickness of $9 \leq t \leq 25 \mathrm{~mm}$, made of SKK490 material as Electric Resistance Welded (herein after ERW) pipes. Young's modulus of the steel is set to $E=200 \mathrm{GPa}$ and Poisson's ratio $\mu=0.3$.

The length of a pier is satisfied with the slenderness ratio of $l / r \leq 120\left(I=K I_{e}\right.$, $K=1$ = effective length factor, $l_{e}=$ length of a pier $h, r=$ cross-section secondary radius). The slenderness ratio parameter $\bar{\lambda}$ and diameter-thickness ratio parameter $R_{t}$ satisfy the range of the following Formulas (1) and (2). This is the range of application of pier member for satisfying the predetermined seismic performance shown in Japan Standard ([4]; herein after Japan Standard V) P228.

$$
\begin{gathered}
0.2 \leq \bar{\lambda}=\frac{1}{\pi} \sqrt{\frac{\sigma_{y}}{E}} \frac{l}{r} \leq 0.4 \\
0.03 \leq R_{t}=\frac{R}{t} \frac{\sigma_{y}}{E} \sqrt{3\left(1-\mu^{2}\right)} \leq 0.08
\end{gathered}
$$

Herein, $\pi$ is the circumference ratio; $\sigma_{y}$ is the yield stress of material.

As it conforms to the Japan Standard IV, the length of pile was set at $10 \mathrm{~m}$, based on $\beta L_{e}>3$ ( $\beta=$ characteristic value calculated Equation (6), $L_{e}=$ length of a pile $L$ ), a semi-infinite-length pile. The ground rigidity is $10 \mathrm{MN} / \mathrm{m}^{2} \leq \alpha E_{0} \leq$ $300 \mathrm{MN} / \mathrm{m}^{2}$. The test cases are shown in Table 1 in the form of PP $h(\mathrm{~m})-$ $t(\mathrm{~mm})-\alpha E_{0}\left(\mathrm{MN} / \mathrm{m}^{2}\right)$.

\begin{tabular}{|c|c|c|c|c|c|c|c|c|c|c|c|c|c|c|}
\hline \multirow{3}{*}{ CASE } & \multicolumn{3}{|c|}{$h(\mathrm{~m}) / L(\mathrm{~m})$} & \multicolumn{4}{|c|}{ diameter and thickness (mm) } & \multicolumn{7}{|c|}{ ground rigidity $\alpha E_{0}\left(\mathrm{MN} / \mathrm{mm}^{2}\right)$} \\
\hline & \multirow{2}{*}{$1 / 10$} & \multirow{2}{*}{$5 / 10$} & \multirow{2}{*}{$10 / 10$} & \multicolumn{4}{|c|}{ D500 } & \multirow{2}{*}{10} & \multirow{2}{*}{50} & \multirow{2}{*}{100} & \multirow{2}{*}{150} & \multirow{2}{*}{200} & \multirow[b]{2}{*}{250} & \multirow[b]{2}{*}{300} \\
\hline & & & & $t=9$ & $t=14$ & $t=19$ & $t=25$ & & & & & & & \\
\hline PP1-9-* & o & & & 0 & & & & o & ० & o & o & o & O & o \\
\hline PP1-14-* & o & & & & o & & & o & o & O & o & ० & o & o \\
\hline PP1-19-* & O & & & & & ० & & ० & ○ & ० & $\circ$ & O & $\circ$ & $\circ$ \\
\hline PP1-25-* & o & & & & & & o & o & o & O & ○ & O & ० & ० \\
\hline PP5-9-* & & O & & $\circ$ & & & & ० & $\circ$ & ० & $\circ$ & O & $\circ$ & $\circ$ \\
\hline PP5-14-* & & o & & & ० & & & o & $\circ$ & o & ० & o & o & ○ \\
\hline PP5-19-* & & ० & & & & $\circ$ & & $\circ$ & $\circ$ & ० & $\circ$ & ० & ० & $\circ$ \\
\hline PP5-25-* & & O & & & & & $\circ$ & $\circ$ & $\circ$ & $\circ$ & $\circ$ & ○ & ○ & $\circ$ \\
\hline PP10-9-* & & & o & ० & & & & ० & ○ & $\circ$ & ० & o & O & $\circ$ \\
\hline PP10-14-* & & & ० & & ० & & & ० & ○ & $\circ$ & ० & o & ० & $\circ$ \\
\hline PP10-19-* & & & ० & & & $\circ$ & & ० & $\circ$ & ० & ० & o & ० & $\circ$ \\
\hline PP10-25-* & & & o & & & & o & O & o & O & 0 & 0 & O & ○ \\
\hline
\end{tabular}

Table 1. Test cases. 


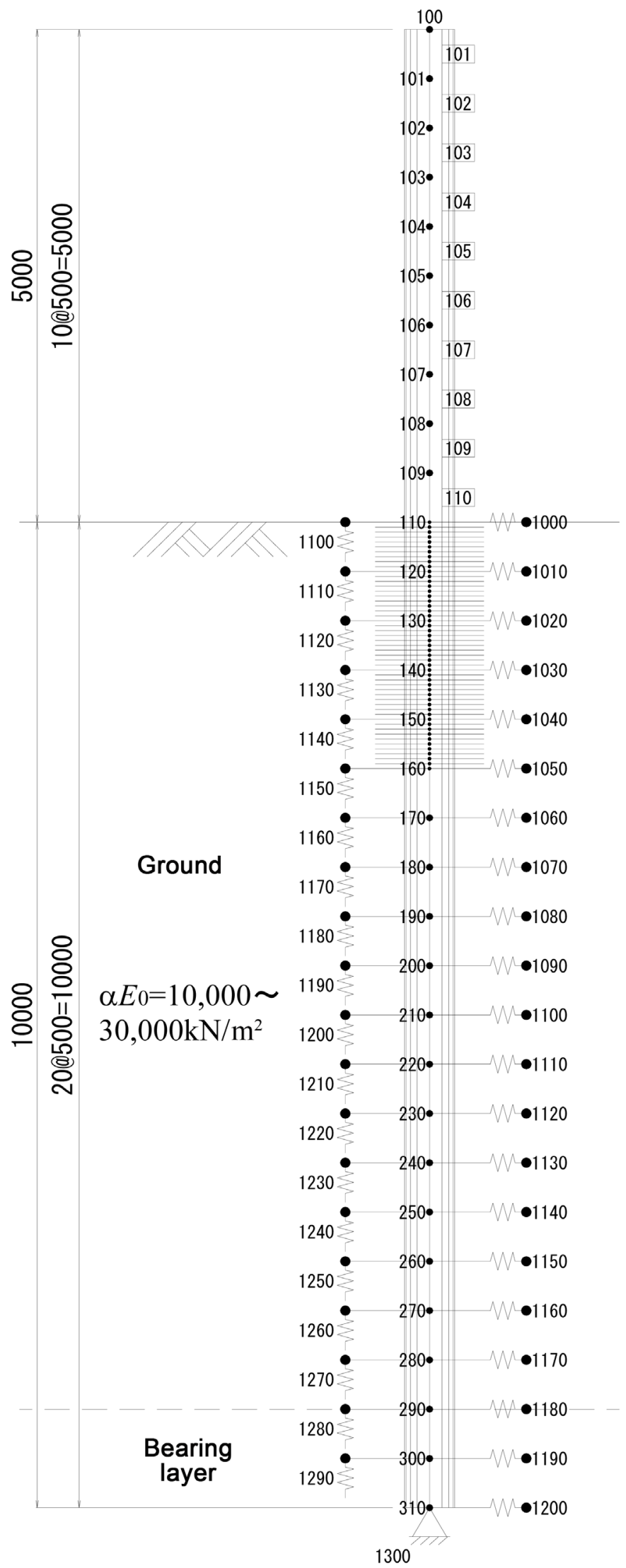

Figure 2. A single column pier with lower part supported by spring. 


\section{Analysis Model and Method}

The analysis model is shown in Figure 2.

A hinged end is adopted as the boundary conditions of a pile tip. The pile part is installed with ground springs in horizontal and vertical directions. The ground spring is calculated by the formula based on the Japan Standard IV PP285-286. These equations were suggested by past experiments and analysis [5] [6] [7], have been adopting in the case of designing the pile foundation of road bridges.

$$
\begin{gathered}
k_{H}=k_{H 0}\left(\frac{B_{H}}{0.3}\right)^{-3 / 4} \\
k_{H 0}=\frac{1}{0.3} \alpha E_{0} \\
B_{H}=\sqrt{D / \beta} \\
\beta=\sqrt[4]{k_{H} D / 4 E I}
\end{gathered}
$$

Herein $k_{H}=$ horizontal subgrade reaction coefficient, $k_{H 0}=$ horizontal subgrade reaction coefficient corresponding to the value of the plate loading test, $B_{H}=\mathrm{A}$ is the conversion loading width of the foundation, and $E I=$ the flexural rigidity of the steel pipe.

It is confirmed that the mechanical properties of ERW (Electric Resistance Welded) pipes are higher in yield ratio than the SM material, and the secondary gradient after yield is small [8] [9] [10] [11]. This study focuses on the rigidity relationship between the ground and the piles and the entire buckling phenomenon in the elastic region according to the effective buckling length. Therefore, the mechanical property of this material is as shown in Figure 3; the yield stress is set to $315 \mathrm{~N} / \mathrm{mm}^{2}$, and secondary gradient is level.

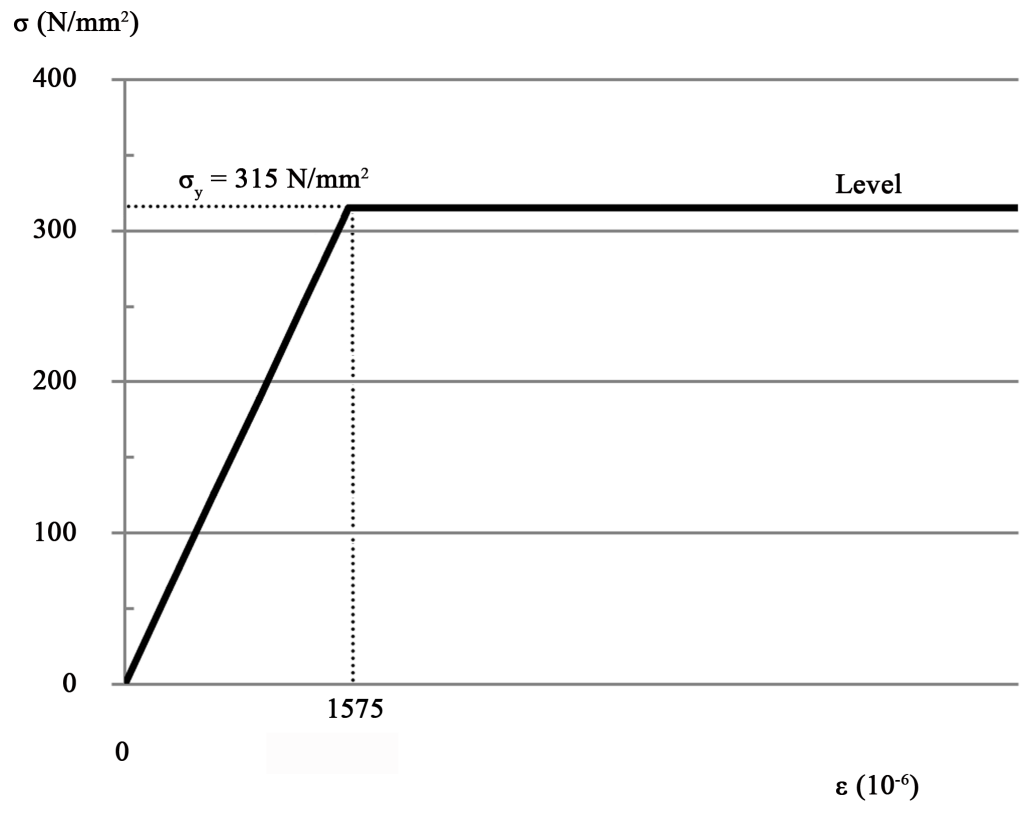

Figure 3. Stress strain curve. 
Effective buckling length $l_{c r}$ is determined from Euler's equation using the primary eigenvalue $\omega$ which is obtained by the eigenvalue analysis. In the analysis, an axial force of $N=1 \mathrm{kN}$ is added to the head of the pier.

$$
l_{c r}=\pi \sqrt{\frac{E I}{\omega N}}
$$

\section{The Relationship between the Characteristic Value Beta and the Effective Buckling Length of the Pier}

The effect that the rigidity difference between the ground and the pile has on the effective buckling length $I_{c r}$ is shown in Figure 4: Relationship between the effective buckling length and $\beta$. The vertical axis shows the dimensionless value resulting from dividing the effective buckling length $l_{c r}$ by the effective buckling length $l_{c r}^{\prime}=2 h$ when the lower end is fixed. The horizontal axis shows the characteristic value $\beta$ indicating the relative relationship between rigidity of the soil and pile rigidity.

From Figure 4, regarding each pier length $1 \mathrm{~m}, 5 \mathrm{~m}, 10 \mathrm{~m}$ respectively, it can be confirmed that as $\beta$ increases, namely due to the increase in rigidity of the soil compared with pile rigidity, the effective buckling length can be confirmed to approach $l_{c r}^{\prime}$. Further, a blue line, which is indicating shorter pier length compared with whole length of Pier-Pile integral structure, is influenced from $\beta$ variation compared with other lines.

In next, due to investigate the relationship with pier length $h$, the horizontal axis of Figure 4 is replaced to $\beta h$ which is obtained by multiplying $\beta$ by $h$. $\beta h$ is a dimensionless value indicating the ratio of $h$ for $\beta$. It is shown in Figure 5: Relationship between the effective buckling length and $\beta h$.

From Figure 5, it can be confirmed that as $\beta h$ increases, the effective buckling length can be confirmed to approach $l_{c r}^{\prime}$. Especially, the relationship of effective buckling length $I_{c r}$ and $\beta h$ is represented as a continuous curve independent of the pier length and thickness. From the above, the effective buckling length $I_{c r}$ of Pier-Pile integral structures is confirmed to be represented by the function of $\beta h$.

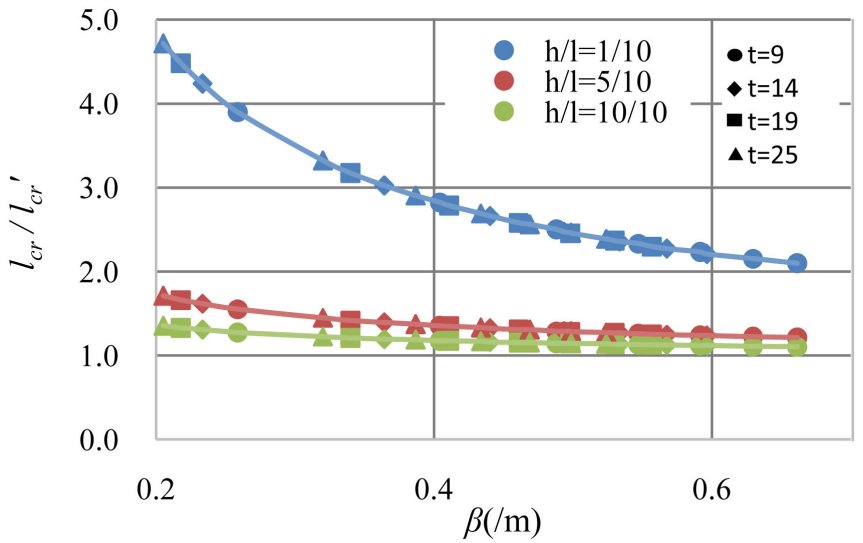

Figure 4. Relationship between the effective buckling length and $\beta$. 


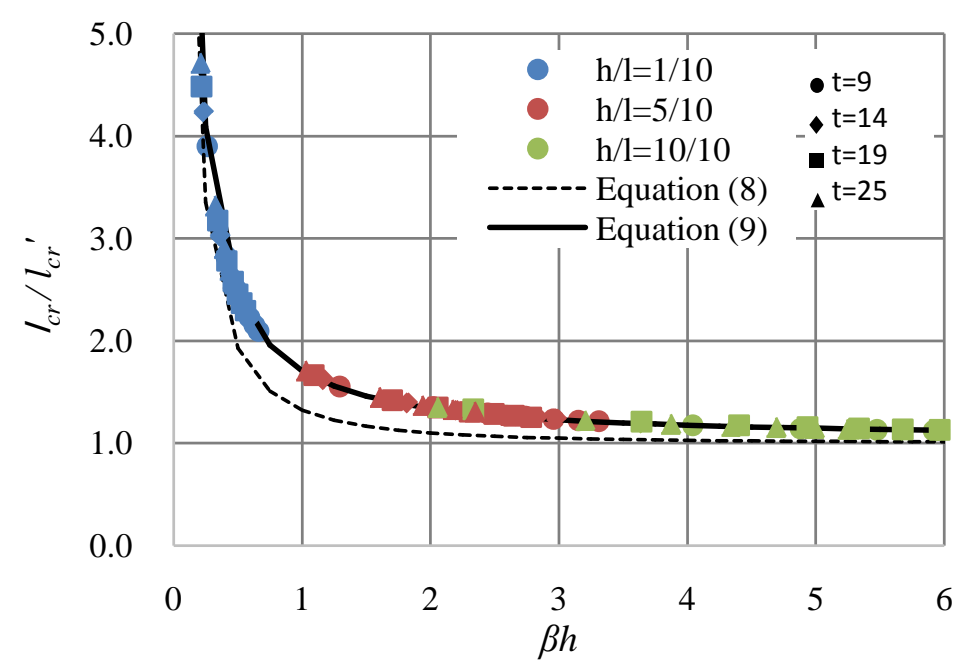

Figure 5. Relationship between the effective buckling length and $\beta$.

Regarding physical meaning of $\beta h, \beta$ is the relative flexural rigidity of Beams on Elastic Foundation, the influence of $\beta$ on the effective buckling length decreases as the value of $\beta h$ increases. Therefore, $\beta h$ is indicating the influence rate for the effective buckling length of Pier-Pile Integral Steel Structure.

\section{Estimation Formula of Effective Buckling Length}

Effective buckling length of a pier with fixed supports is represented by $2 h$ when the pier length is $h$. In addition, it is consistent with the point of $M_{\max }$, the position of the fixed point, and a deflection angle of 0 points. Figure 6 shows Effective buckling length of a pier supported by fix.

Whereas, in the case of a pier supported by spring, effective buckling length differs from the point of $M_{\max }$ and the deflection angle of 0 points. This is because a pile is displaced in the land surface position and deformation modes in the ground are different due to the rigidity difference between the steel pipe and the ground. Figure 7 shows Effective buckling length of a spring supported pier.

It is of note that it is assumed that the buckling mode of the pier is convex in the vicinity of the point of $M_{\max }$ and that effective buckling length of the Pier-Pile integral steel structure is indicated by Equation (8), which is twice the length of Chang equation $I_{m}$ [3] plus $h$.

$$
l_{c r} \fallingdotseq 2\left[h+\frac{1}{\beta} \tan ^{-1}\left\{\frac{1}{(1+2 \beta h)}\right\}\right]=2 h\left[1.0+\frac{1}{\beta h} \tan ^{-1}\left\{\frac{1}{(1+2 \beta h)}\right\}\right]
$$

Figure 5 shows the estimated line of Equation (8). The variation confirmed in Figure 5 is assumed to be due to the accuracy of analysis $\alpha_{l}$, and the correlation factor $\alpha_{2}$ between the dimensionless quantity $\beta h$ that affect the deformation mode. Therefore, Equation (8) can be modified to Equation (9) using the analysis accuracy factor $\alpha_{1}$ and the correlation factor $\alpha_{2}$. Herein when the factors are given as $\alpha_{1}=1.05$ and $\alpha_{2}=0.1$, the estimated line of (9) is consistent with the analytical value of Figure 5 . 

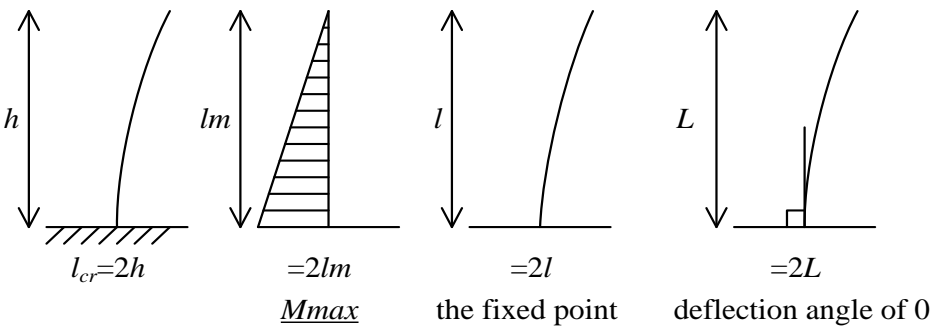

Figure 6. Effective buckling length of a pier supported by fix.

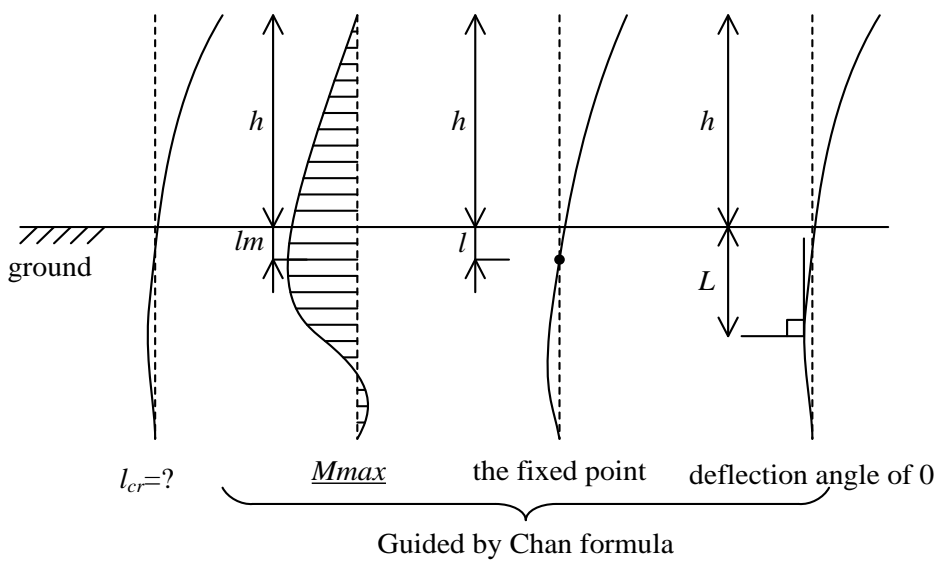

Figure 7. Effective buckling length of a spring supported pier.

It should be noted that the correlation function is 0.999 , and the standard deviation is 0.042 .

$$
k=\frac{l_{c r}}{h}=2 \alpha_{1}\left[1.0+\frac{1}{\beta h} \tan ^{-1}\left\{\frac{1}{\left(1+2 \alpha_{2} \beta h\right)}\right\}\right]
$$

Herein $k$ indicates the ratio of the effective buckling length $l_{c r}$ to pier height $h$, $k$ would be 2.0 when the pier bottom is fixed.

\section{Conclusions}

The following item was revealed in this study.

- Effective buckling length $l_{c r}$ of Pier-Pile integral steel structures can be defined by the simple formula $\beta$.

- $\beta h$ is indicating the influence rate of the effective buckling length of Pier-Pile Integral Steel Structure.

- Newer concept of effective buckling length ratio $k$ was developed.

It should be considered the applicable range of proposed formula. Especially, the ground spring was proposed to be an elastic region under the condition which is assumed that it is mainly used within a displacement of about $1 \%$ of the pile diameter. Therefore, from now on, it is planned to investigate regarding the accuracy of the ground rigidity depending on the allowable displacement amount.

Moreover, for Pier-Pile integral steel structures, it was indicated the possibility for utilization of the existing load-bearing capacity curve based on the slenderness 
ratio parameter using $\beta$. As next step, it will be planned to investigate the load bearing capacity considering the effect of the largeness of the initial condition.

\section{References}

[1] Schlaichbergermann Partner: Award for the Footbridge to Le Mont Saint-Michel in France, 2016.2.

http://www.sbp.de/en/news/award-for-the-footbridge-to-le-mont-saint-michel-in-f rance-1/

[2] SPECIFICATIONS FOR HIGHWAYBRIDGES PART2 STEEL BRIDGES, Japan Road Association, 2014. (In Japanese)

[3] SPECIFICATIONS FOR HIGHWAYBRIDGES PART4 SUBSTRUCTURES, Japan Road Association, 2014. (In Japanese)

[4] SPECIFICATIONS FOR HIGHWAYBRIDGES PART5SEISMIC DESIGN, Japan Road Association, 2014 (In Japanese)

[5] The Modeling of the Horizontal Resistance of the Pile Foundation Subjected to Large Displacement, PWRI Documents Number 4100, Public Works Research Institute, 2008. (In Japanese)

[6] The Study on the Stable Design of Road Bridge Foundation in performance provision system, PWRI documents number 4136, Public Work Research Institute, 2009. (In Japanese)

[7] PILE FOUNDATION DESIGN MANUAL, Japan Road Association, Mar., 2017. (In Japanese)

[8] Aoki, T. and Fukumoto, Y. (1981) Stocastic Material Properties and Estimate of Residual Stresses of Cold-Formed-Welded Steel Tubular Members with Small Diameter. Proceedings of JSCE, No. 314, 39-51. (In Japanese)

[9] Nishimura, N., Takeuchi, S., Murakami, S. and Sanui, K. (1997) The Effect Manufacturing Processes on Residual Stress and Yield Stress of ERW Pipe. Proceedings of JSSC, No. 4-13. (In Japanese)

[10] Ozoe, H., Ono, K., Nanazawa, T., Kohno, T. and Ohmori, T. (2016) An Experimental Study on Ultimate Strength and Ductility of Electric Resistance Welded Pipes. The 11 th German Japanese Bridge Symposium, Osaka, 30-31 August 2016, 159-160

[11] Iijima, S., Harada, T., Ohmori, T., Ozoe, H., Kohno, T. Nanazawa, T. and Ono, K. (2015) A Study on Seismic Evaluation Methods for Frame Structures in Multi-Pile Style without Footing. Proceedings of the 18th Symposium on Performance-based Seismic Design Method for Bridges, Tokyo, 7-8 July 2015, 351-356. (In Japanese) 